\title{
Bloch surface waves, a 2D platform for planar optical integration
}

\author{
H. P. Herzig, E. Barakat, L. Yu, R. Dubey \\ Optics \& Photonics Technology Laboratory (OPT) \\ Ecole Polytechnique Fédérale de Lausanne (EPFL), Neuchâtel, Switzerland.
}

\begin{abstract}
A novel platform suitable for fundamental investigations of light propagation through micro- and nanostructures will be discussed. This platform is based on a dielectric multilayer that sustains Bloch surface waves (BSWs). BSWs are electromagnetic surface waves excited at the interface between a truncated periodic dielectric multilayer and a surrounding media. The modification of the top surface to customize a complete 2D micro-system can be produced using e-beam writing, optical lithography or other patterning techniques. The results obtained confirm the possibility of developing a robust multilayer platform that would pave the way for integration of photonic components in photonic chips using standard waferscale production.
\end{abstract}

Keywords-micro-optics; microsystem; Bloch survace waves, $2 D$ platform; nanophotonics;

\section{INTRODUCTION}

Carefully designed periodic dielectric structures sustain surface electromagnetic states, called Bloch surface waves (BSWs), within their photonic bandgaps [1]. There are several advantages provided by BSW propagation. First of all, due to the use of dielectric material absorption, losses are very low when transparent materials are considered at the desired wavelength. This allows the propagation of BSWs over long distances and thus, the spectral range is not limited anymore by the material properties. Another advantage of using BSWs is the possibility of its excitation with any wavelength by optimizing either the multilayer thicknesses or the incident beam angle. Furthermore, because the maximum intensity associated with the BSW can be tuned on the surface, a strong field intensity, increased by several orders of magnitudes, can be achieved and thereby enhance the light-matter interaction close to the surface [2].

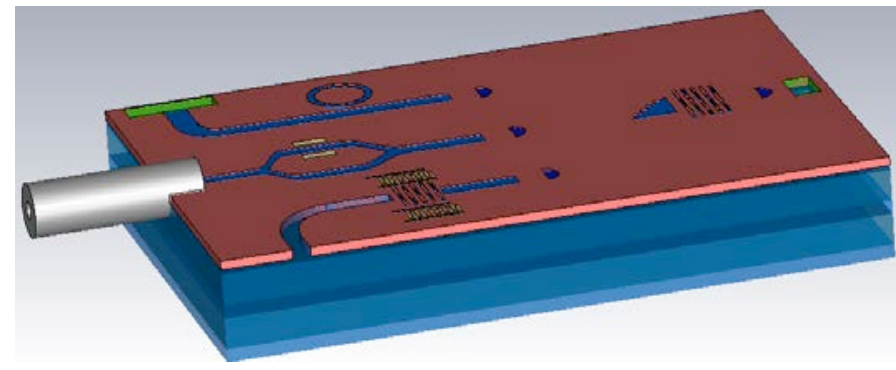

Fig. 1. BSW platform showing a system with different components to propagate Bloch surface waves (BSWs).
We propose and demonstrate that a BSW-sustaining multilayer is a suitable general platform for manipulating and control the BSWs propagation on its surface in the spectral range of telecom wavelengths.

\section{CONCEPT OF THE BSW PlatForm}

Several 2D optical photonic devices can be implemented starting from an ultra-thin $(\approx \lambda / 15)$ additional layer deposited on the multilayer that can be subsequently shaped as desired [3]. One of the main advantages is that these 2D photonic devices can have arbitrary shapes, which is difficult to obtain in 3D. Since BSWs can be considered as a $2 \mathrm{D}$ wave and it is bounded at the surface of the multilayer, near-field imaging is one of the preferred tools to directly monitor and characterize the nearfield produced on the structured multilayered surface. Multiheterodyne scanning near-field optical microscope is used in the near-field characterization [4]. The modification of the top surface to customize a 2D micro-system can be produced, e.g., by e-beam writing, optical lithography, stamping and other replication techniques.

Fig. 1 shows the basic concept of the BSW platform. Our recent results demonstrated the possibility of developing a robust multilayer platform that would pave the way for integration of photonic components in photonic chips using standard wafer-scale production [5]. Various 2D polymer photonic-components have been realized on top of the platform. We experimentally and theoretically demonstrate that the BSWs can be diffracted, focused, coupled and made resonating by locally shaping the geometries of $2 \mathrm{D}$ photonic devices on the multilayer, such as lenses, prisms, gratings, bended waveguides and waveguide couplers, etc.

\section{RING RESONATORS}

An important device in an optical microsystem is a ring resonator. In order to prove that such micro-resonators are feasible on the BSW based platform, we numerically construct a disk resonator. A disk resonator is introduced because it has less loss compared with a ring resonator. In fact, the presence of both inner and outer rims of a ring resonator generally increases the scattering losses. Taken the maximum refractive index contrast that one can obtain with the present configuration and material, a model of a micro-disk resonator is constructed in $2 \mathrm{D}$. Note that the following values are not limitations. The value of the refractive index contrast 
corresponds to the difference of the effective indices of the resonator and its surrounding. We present FDTD-computed, results for resonance frequencies and quality factor of diskresonators. It consists of a disk having a radius $\mathrm{R}$ coupled to a straight waveguide of width $\mathrm{w}$, where a fraction $\mathrm{k}$ of the incoming light is transferred to the resonator. The distance separating the disk and the waveguide is defined by the gap g. The computed wavelength response of a non-optimized disk resonator of radius $\mathrm{R}=40 \mu \mathrm{m}$, width $\mathrm{w}=2 \mu \mathrm{m}$ and a gap of $300 \mathrm{~nm}$ is shown in Fig. 2. Periodic fringes appear in the wavelength response at the output port; the additional resonance corresponds to the higher-order whispering gallery modes. The quality factor $\mathrm{Q}$ is directly deduced from the transmission spectrum $(\mathrm{Q} \sim \lambda / \Delta \lambda)$.

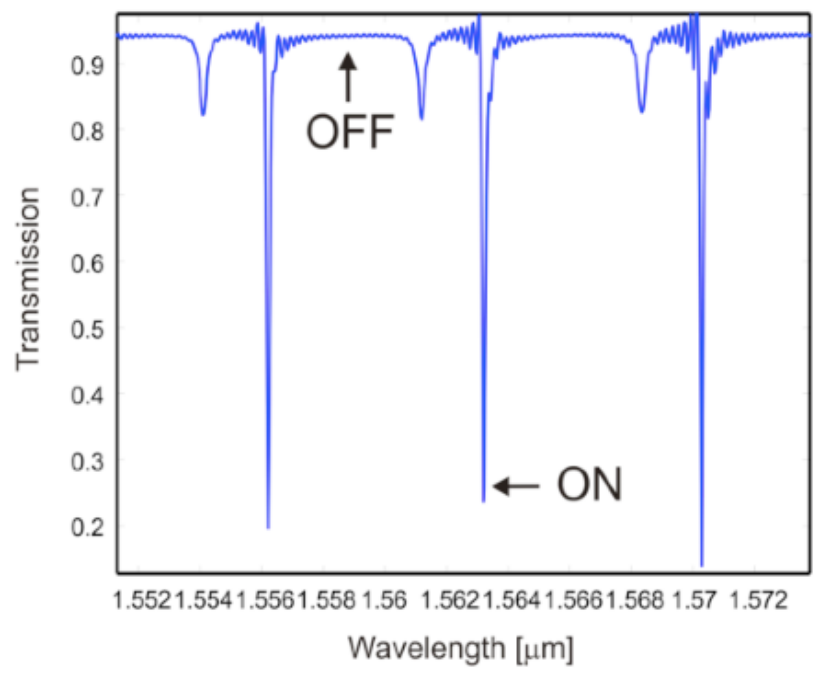

Fig. 2. Wavelength response (transmission) of the resonator.

We obtained a factor of $2 \times 10^{4}$ where $18 \%$ of the incident light is coupled to the disk. A key mechanism that affects the quality factor is the coupling coefficient between the cavity and the adjacent waveguide.

$$
Q=\frac{2 \pi R n_{e f f}}{\lambda} \frac{\pi}{k^{2}} .
$$

$\mathrm{k}$ and $\mathrm{Q}$ strongly depend on the refractive index contrast, $\mathrm{Q}$ remains higher for weaker coupling. $(\mathrm{ON}, \lambda=1563.2 \mathrm{~nm})$ and out of the resonance (OFF, $\lambda=1559.15 \mathrm{~nm}$ ).

In Fig. 3, we display the effect of varying both the gap between the straight waveguide and the ring radius on the coupling efficiency and the quality factor. The refractive index of the input bus and the ring are considered the same (TiO2). The waveguide width is $2 \mu \mathrm{m}$. First in Fig. 6(a) we show the coupling efficiency and the quality factor as function of the gap size. A well-known phenomenon is seen, the coefficient factor decreases by increasing the gap value, however the quality factor increases with the gap because it is inversely proportional to the coupling coefficient. On the other hand, the quality factor increases (Fig. 6(b)) by increasing with radius value matching the FDTD simulations done previously.

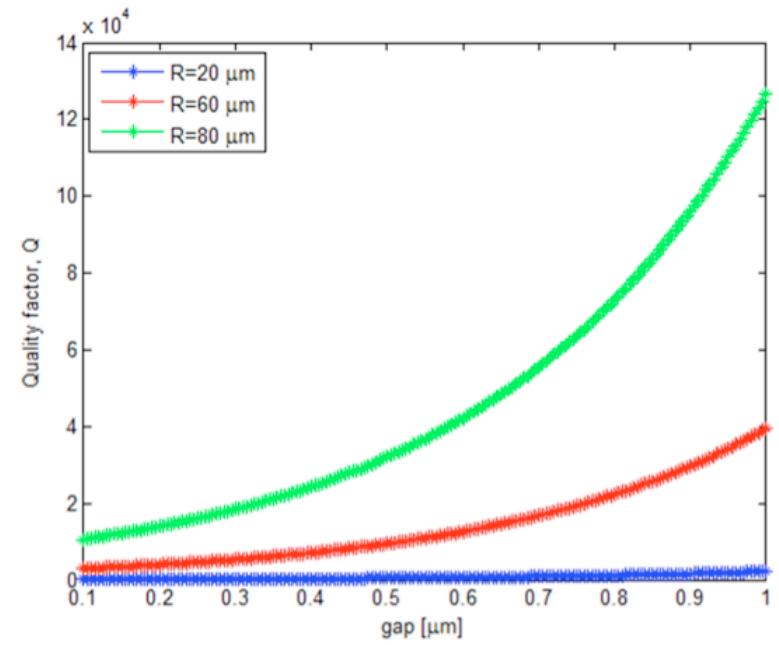

(a)

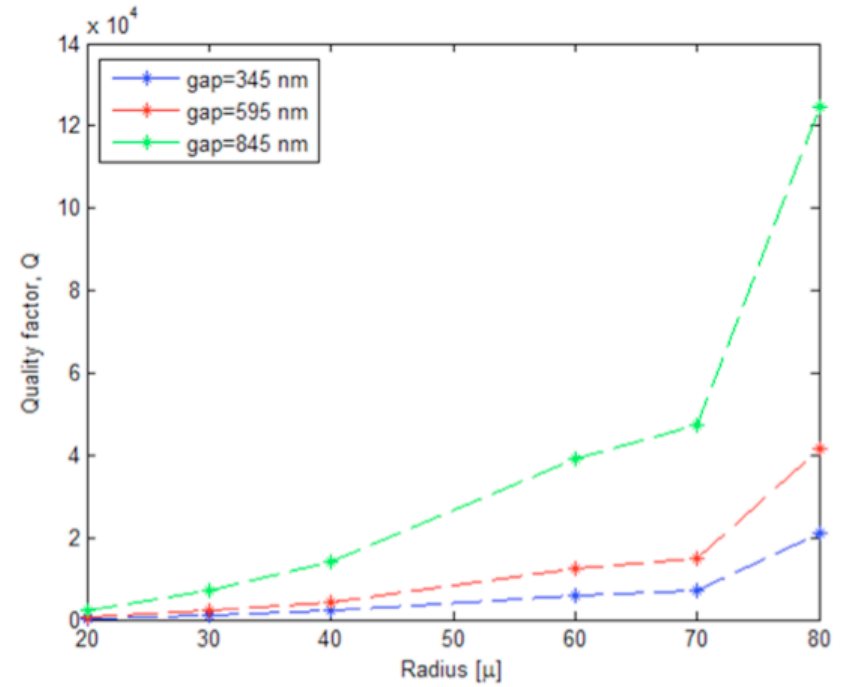

(b)

Fig. 3. (a) Coupling efficiency as a function of the gap; (b) Quality factor $Q$ versus ring radius.

\section{REFERENCES}

[1] P. Yeh, A. Yariv, A. Y. Cho, "Optical surface waves in periodic layered media," Appl. Phys. Lett. 32, 104-105 (1978).

[2] E. Descrov, T. Sfez, L. Dominici, W. Nakagawa, F. Michelotti, F. Giorgis, H. P. Herzig, "Near-field imaging of Bloch surface waves on silicon nitride one-dimensional photonic crystals," Opt. Express 16, 5453-5464 (2008).

[3] E. Descrovi, T. Sfez, M. Quaglio, D. Brunazzo, L. Dominici, F. Michelotti, H. P. Herzig, O. J. F. Martin, F. Giorgis, "Guided Bloch Surface Waves on Ultrathin Polymeric Ridges," Nano Lett. 10, 2087 2091 (2010).

[4] L. Yu, T. Sfez, V. Paeder, P. Stenberg, W. Nakagawa, M. Kuittinen, and H. P. Herzig, "Concurrent polarization retrieval in multi-heterodyne scanning near-field optical microscopy: validation on silicon formbirefringent grating," Opt. Express 20, 23088-23099 (2012).

[5] L. Yu, E. Barakat, T. Sfez, L. Hvozdara, J. Di Francesco, and H. P. Herzig, "Manipulating Bloch surface waves in 2D: a platform conceptbased at lens," Light: Science \& Applications 3, e124 (2014). LSA 2014 